2016

\title{
Auxiliary-field quantum Monte Carlo calculations of the molybdenum dimer
}

Wirawan Purwanto

College of William and Mary, Dept Phys, Williamsburg, VA 23187 USA

Shiwei Zhang

College of William and Mary, Dept Phys, Williamsburg, VA 23187 USA

Henry Krakauer

College of William and Mary, Dept Phys, Williamsburg, VA 23187 USA

Wirawan Purwanto

Old Dominion Univ, Norfolk, VA 23529 USA

Follow this and additional works at: https://scholarworks.wm.edu/aspubs

\section{Recommended Citation}

Purwanto, W., Zhang, S., \& Krakauer, H. (2016). Auxiliary-field quantum Monte Carlo calculations of the molybdenum dimer. The Journal of chemical physics, 144(24), 244306.

This Article is brought to you for free and open access by the Arts and Sciences at W\&M ScholarWorks. It has been accepted for inclusion in Arts \& Sciences Articles by an authorized administrator of W\&M ScholarWorks. For more information, please contact scholarworks@wm.edu. 


\section{Auxiliary-field quantum Monte Carlo calculations of the molybdenum dimer}

Cite as: J. Chem. Phys. 144, 244306 (2016); https://doi.org/10.1063/1.4954245

Submitted: 28 March 2016 . Accepted: 08 June 2016 . Published Online: 27 June 2016

Wirawan Purwanto (D), Shiwei Zhang, and Henry Krakauer

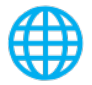

\section{ARTICLES YOU MAY BE INTERESTED IN}

An auxiliary-field quantum Monte Carlo study of the chromium dimer

The Journal of Chemical Physics 142, 064302 (2015); https://doi.org/10.1063/1.4906829

Excited state calculations using phaseless auxiliary-field quantum Monte Carlo: Potential energy curves of low-lying $\mathrm{C}_{2}$ singlet states

The Journal of Chemical Physics 130, 094107 (2009); https://doi.org/10.1063/1.3077920

Assessing weak hydrogen binding on $\mathrm{Ca}^{+}$centers: An accurate many-body study with large basis sets

The Journal of Chemical Physics 135, 164105 (2011); https://doi.org/10.1063/1.3654002

Where in the world is AIP Publishing? Find out where we are exhibiting next 


\title{
Auxiliary-field quantum Monte Carlo calculations of the molybdenum dimer
}

\author{
Wirawan Purwanto, ${ }^{\text {a) }}$ Shiwei Zhang, and Henry Krakauer \\ Department of Physics, College of William and Mary, Williamsburg, Virginia 23187-8795, USA
}

(Received 28 March 2016; accepted 8 June 2016; published online 27 June 2016)

\begin{abstract}
Chemical accuracy is difficult to achieve for systems with transition metal atoms. Third row transition metal atoms are particularly challenging due to strong electron-electron correlation in localized $d$-orbitals. The $\mathrm{Cr}_{2}$ molecule is an outstanding example, which we previously treated with highly accurate auxiliary-field quantum Monte Carlo (AFQMC) calculations [W. Purwanto et al., J. Chem. Phys. 142, 064302 (2015)]. Somewhat surprisingly, computational description of the isoelectronic $\mathrm{Mo}_{2}$ dimer has also, to date, been scattered and less than satisfactory. We present high-level theoretical benchmarks of the $\mathrm{Mo}_{2}$ singlet ground state $\left(X^{1} \Sigma_{g}^{+}\right)$and first triplet excited state $\left(a^{3} \Sigma_{u}^{+}\right)$, using the phaseless AFQMC calculations. Extrapolation to the complete basis set limit is performed. Excellent agreement with experimental spectroscopic constants is obtained. We also present a comparison of the correlation effects in $\mathrm{Cr}_{2}$ and $\mathrm{Mo}_{2}$. Published by AIP Publishing. [http://dx.doi.org/10.1063/1.4954245]
\end{abstract}

\section{INTRODUCTION}

Transition metal (TM) dimers are of special interest theoretically and computationally. They fully exhibit the complexity of more complex TM materials (such as the formation of high-order bonds), but their relatively small sizes make them amenable to systematic and rigorous theoretical studies. Group VIB dimers are especially interesting, because the atom fragments are in the high-spin state $\left({ }^{7} \mathrm{~S}\right)$, and they form a closed shell $\left({ }^{1} \Sigma\right)$ configuration in the molecular ground state. This results in a many-body spectrum with many nearly degenerate states, with strong electronic correlation effects. The $\mathrm{Mo}_{2}$ molecule is similar to $\mathrm{Cr}_{2}$ in that both are highly multiconfigurational in nature and require accurate treatment of both static and dynamic electron correlation. Because the $4 \mathrm{~d}$ orbitals are not as localized as $3 \mathrm{~d}$, the severity of electronic correlation is significantly reduced for $\mathrm{Mo}_{2}$. For example, a complete active space self-consistent field (CASSCF) treatment in $\mathrm{Mo}_{2}$ recovers about $30 \%$ of the experimental binding energy, while with the same wave function, $\mathrm{Cr}_{2}$ is not even bound. Nevertheless, the best quantum chemistry calculations for $\mathrm{Mo}_{2}$ give widely varying predictions. ${ }^{1-3}$

In this work, we present accurate theoretical calculations of $\mathrm{Mo}_{2}$ potential energy curves (PECs) near the equilibrium geometry. We consider both the singlet ground state $\left(X^{1} \Sigma_{g}^{+}\right)$and triplet first excited state $\left(a^{3} \Sigma_{u}^{+}\right)$, and calculate their spectroscopic properties. We employ the phaseless auxiliary-field quantum Monte Carlo (AFQMC) method $^{4-7}$ in our calculations. Select benchmark calculations were also performed with exact free-projection (FP) $\mathrm{AFQMC}^{7-9}$ to help establish the accuracy of our calculations. A high-quality quadruple zeta (QZ) basis set is used for the majority of our calculations. Extrapolation to the complete basis set (CBS) limit is performed in combination with the triple zeta (TZ)

\footnotetext{
a)Present address: Old Dominion University, Norfolk, Virginia 23529, USA. Electronic mail: wirawan0@gmail.com
}

basis results, following standard approaches. As shown below, the AFQMC results for the spectroscopic constants are in excellent agreement with experiment. We compare our results with those from other quantum chemistry approaches. The similarities and differences in electron-electron correlation between $\mathrm{Mo}_{2}$ and $\mathrm{Cr}_{2}$ are also examined.

The remainder of the paper proceeds as follows. The methodology is discussed in Section II. Results of our $\mathrm{Mo}_{2}$ calculations are presented in Section III. Section IV presents comparisons with previous many-body quantum chemistry results and an analysis of the relative sizes of the correlation energy contributions in $\mathrm{Mo}_{2}$ and $\mathrm{Cr}_{2}$, as well as the effect of the trial wave function in the AFQMC calculations. We summarize our results in Section V.

\section{METHODOLOGY}

The AFQMC method projects the many-body ground state wave function from a given trial wave function. ${ }^{10-12}$ It is implemented as random walks of Slater determinants, with orbitals expressed in a chosen single-particle basis. A phaseless approximation ${ }^{4}$ has been introduced to control the phase problem introduced by complex auxiliary-field sampling, resulting in a practical computational method that scales modestly with the system size [e.g., $O\left(N^{3}\right)$ or $O\left(N^{4}\right)$ ]. Its high accuracy has been demonstrated in many molecular and solid systems ${ }^{5,8,13-16}$ as well as model electronic systems. ${ }^{7,17}$

As an orbitally based wave function method, the AFQMC theoretical framework has close relations to many-body quantum chemistry methods. When expressed in a oneparticle gaussian type orbital (GTO) basis, both approaches use exactly the same Hamiltonian. Thus, many efficient techniques developed for correlated quantum chemistry methods can be directly imported. This was done, for example, by using Cholesky decomposition to remove a 
bottleneck in the handling of two-body interaction matrix elements for large basis sets ${ }^{18}$ and introducing frozencore type of approaches to seamlessly embed the highly correlated AFQMC treatment within a larger mean-field environment. ${ }^{14,16}$

Since static correlation is important in $\mathrm{Mo}_{2}$, it is desirable that the trial wave function accounts for this effect well. We perform CASSCF(12e,12o) calculations, which correlate 12 electrons in 12 active orbitals derived from $4 \mathrm{~d}$ and $5 \mathrm{~s}$ atomic states. The resulting multideterminant expansion is then truncated to retain about $93 \%-95 \%$ of the total weight, yielding a multi-determinant wave function which is used without further optimization as our trial wave function. Typically this gives a trial wave function with 90-240 determinants for the ground state, and $30-290$ for the excited state. Since the number of determinants grows rapidly with bond stretching, we have to use a shorter cutoff (86\%-92\%) at larger bond lengths $(\geq 2.2 \AA)$ in order to keep the number of determinants in a range that is easy to handle with the current state of our code.

We perform our AFQMC calculations using the all-electron atomic natural orbital relativistic correlation consistent (ANO RCC) GTO basis. ${ }^{19}$ Scalar relativity is treated with the Douglas-Kroll-Hess Hamiltonian. Spin-orbit effects on the dissociation energy were assessed with density functional theory calculations, using NWCHEM, with a cc-pwCVTZ-PP basis, and Dolg's energy-consistent spinorbit relativistic effective core potential (ECP). There was essentially no effect on the dissociation energy (with the spinorbit generalized gradient approximation PBE functional), so this was not pursued further. The $\mathrm{Ar}+3 \mathrm{~d}^{10}$ atom-like core orbitals are frozen at the mean-field (CASSCF) level. The calculated results were obtained using basis sets up to the realistic QZ (8s7p5d3f2g1h contraction) basis, ${ }^{3}$ denoted ANO-QZ hereafter. As shown below, this basis provides excellent results for many quantities. The exception is the binding energy, which needs to be extrapolated to the CBS limit in order to make reliable comparisons with experiment.

\section{RESULTS}

We first present $\mathrm{Mo}_{2}$ AFQMC results obtained with the ANO-QZ basis, before discussing the CBS-extrapolated results. Previously reported many-body calculations ${ }^{1-3}$ largely used multireference perturbative methods. The ANO-QZ CASPT2 (complete active space second-order perturbation theory) results of Borin et al. ${ }^{3}$ are among the most accurate. The exact and approximate AFQMC results, using the same basis, will provide a useful benchmark to these results.

Figure 1 shows the $\mathrm{Mo}_{2} \mathrm{AFQMC}$ binding-energy curves of the ground (singlet $X$ ) and excited (triplet $a$ ) states in the ANO-QZ basis. (The binding energy is defined as the difference between the molecular total energy and that of the two isolated atoms. It is shown as a function of $R_{\mathrm{Mo}-\mathrm{Mo}}$, the distance between the two $\mathrm{Mo}_{2}$ nuclei.) AFQMC calculations of the Mo atoms were done using the ROHF trial wave function. For the ground state of the molecule,

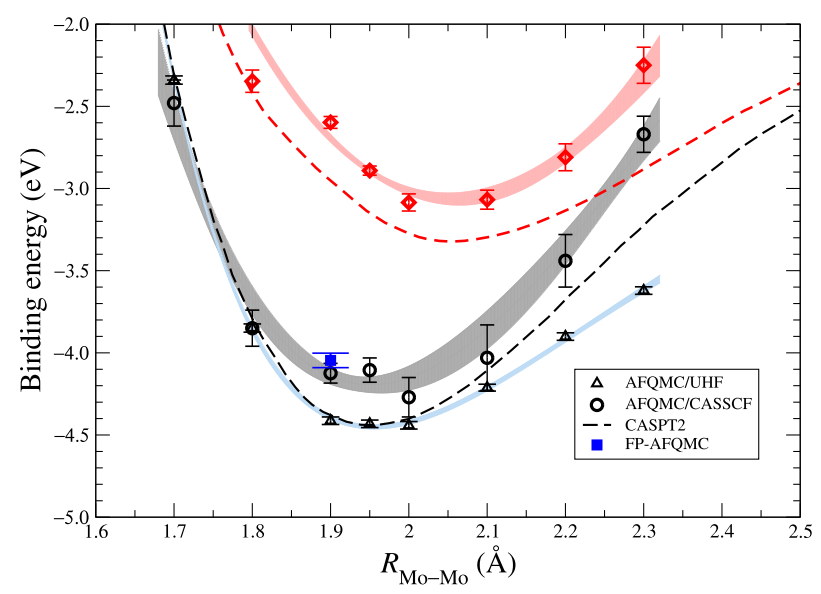

FIG. 1. AFQMC and CASPT2 $\mathrm{Mo}_{2}$ PECs, both using the ANO-QZ basis, for the ground state (dark colors) and the lowest-energy triplet excited state (red). Shaded curves are Morse fits to the AFQMC results. CASPT2 PECs from Ref. 3 are shown as dashed lines. Ground-state AFQMC: AFQMC/UHF results are shown as triangle symbols with error bars and light blue shading; AFQMC/CASSCF are shown as black circles and grey shading. Only AFQMC/CASSCF results are shown for the excited state (red diamonds and red shading). Exact FP-AFQMC result for the ground state at $R(\mathrm{Mo}-\mathrm{Mo})$ $=1.9 \AA$ is shown by the blue square.

phaseless AFQMC calculations are performed using both UHF (AFQMC/UHF) and truncated CASSCF (AFQMC/CASSCF) trial wave functions. The computed binding energy curves are shown; also shown is the exact free projection AFQMC (FP$\mathrm{AFQMC}$ ) binding energy for a geometry near equilibrium. For the excited state, only AFQMC/CASSCF results are shown. Morse curves are fitted to these results and are shown as color bands whose width represents the combined stochastic and fitting uncertainties. The ground state appears to exhibit stronger correlations, as evidenced by the larger statistical error bar for similar quality of trial wave functions and roughly comparable amounts of AFQMC computation. This is also consistent with the observation that the Hartree-Fock energy for the singlet state is higher than the triplet and that a larger number of determinants are in the trial wave function for the ground state even though the same cutoff is applied when truncating both the ground- and excited-state CASSCF wave functions (see Sec. II).

While AFQMC/UHF overestimates the binding energy by $\sim 0.25 \mathrm{eV}$ compared to the exact FP-AFQMC, AFQMC/CASSCF shows excellent agreement. This establishes the high accuracy of the truncated CASSCF trial wave function for $\mathrm{Mo}_{2}$. Similar behavior was found in our previous work on $\mathrm{Cr}_{2},{ }^{15}$ where a multi-determinant truncated CASSCF trial wave function was also required. In the more strongly correlated $\mathrm{Cr}_{2}$ molecule, however, AFQMC/UHF overestimated the binding by $\sim 0.9 \mathrm{eV}$ near the equilibrium bond length for a TZ basis. ${ }^{15}$ For comparison, the CASPT2 results $^{3}$ in the same basis set are also shown in Fig. 1. We see that both the ground and excited states appear to be overbound by $\sim 0.3 \mathrm{eV}$. In the ground state of $\mathrm{Cr}_{2}$, CASPT2 results showed the same trend, resulting in overbinding by $\sim 0.8 \mathrm{eV} .{ }^{15,20}$

The CBS-extrapolated AFQMC/CASSCF PECs are shown in Figure 2. As in our previous work, ${ }^{15}$ the $\mathrm{QZ} \rightarrow \mathrm{CBS}$ correction was obtained from AFQMC/UHF calculations 


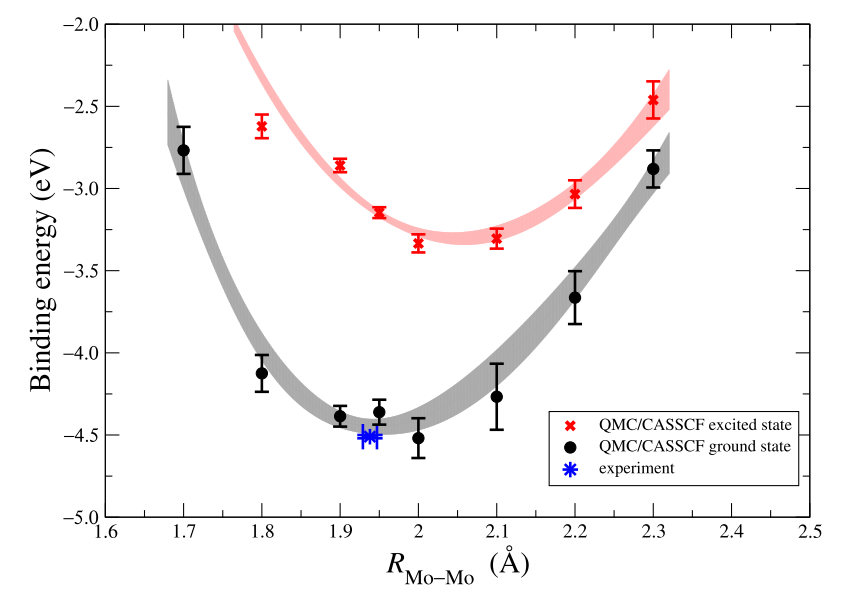

FIG. 2. CBS-extrapolated AFQMC/CASSCF PECs. Symbols, colors, and shading are as in Fig. 1. The ground state experimental binding energy and bond length are given by the blue starred symbol, with the error bars representing the experimental uncertainties. The zero-point energy has been removed from the experimental binding energy.

using the ANO-TZ (7s6p4d2f1g) and ANO-QZ basis sets. Extrapolation with only these two available basis sets is less than ideal and could potentially cause uncertainties. In $\mathrm{Cr}_{2}$, we were able to validate the TZ-QZ extrapolation with explicit calculations using the $5 \mathrm{Z}$ basis set. Cross comparison of the results here with those from $\mathrm{Cr}_{2}$ is valuable and helps to improve confidence in the procedure. We use a two-part scheme to extrapolate the many-body energies to the CBS limit: ${ }^{18}$ the exponential ansat ${ }^{21}$ for the HF energies (with exponent $c=1.63$ ) and the inverse cubic form $^{22}$ for the correlation energies. The QZ $\rightarrow$ CBS correction increases the magnitude of the AFQMC binding energy by about $0.3 \mathrm{eV}$ and $0.2 \mathrm{eV}$ at the shortest and longest bond distances $\left(R_{\mathrm{Mo}-\mathrm{Mo}}=1.7\right.$ and $2.2 \AA$, respectively). In this geometry range, the correction is well approximated by a linear function of $R_{\mathrm{Mo}-\mathrm{Mo}}$. This correction was applied to ANO-QZ AFQMC/CASSCF PECs to obtain the CBS limit. Test calculations showed that the excited state CBS correction was within error bars of the ground state CBS value, so we used the ground state correction for both.

Spectroscopic constants corresponding to Figs. 1 and 2 are given in Tables I and II for the ground and excited states, respectively. The tables also show results from experiment and from other high-level quantum chemistry many-body calculations. Our coupled cluster singles and doubles with perturbative triples $[\mathrm{CCSD}(\mathrm{T})]$ results were extrapolated to the CBS limit following the same procedure as described above, using $\operatorname{CCSD}(\mathrm{T})$ calculations for all basis sets; the CBS correction obtained this way was slightly smaller than, but consistent with, AFQMC/UHF CBS correction. For multireference perturbative calculations, the CBS extrapolation is less straightforward, since their correlation energies do not fit the inverse-cubic ansatz well, as discussed further below. Consequently, we made no attempt to apply the same CBS corrections to the perturbative results in Tables I and II; the values are listed in a separate column and correspond to the specified basis set.

As discussed in connection with Fig. 1, AFQMC/ CASSCF is essentially exact near equilibrium. Thus, the AFQMC/CASSCF results in Tables I and II provide a benchmark for assessing the other quantum chemistry methods. At the CBS limit AFQMC/CASSCF is seen to be in excellent agreement with the experiment. In contrast, other quantum chemistry results show considerable variance, especially for the molecular dissociation energy $D_{e}$. This is discussed further in Sec. IV.

TABLE I. Ground state $\left(X^{1} \Sigma_{g}^{+}\right)$spectroscopic properties of $\mathrm{Mo}_{2}$ computed using phaseless AFQMC and other quantum chemistry methods. $D_{e}$ is the molecular dissociation energy, in units of eV (where the zero-point energy $\sim 0.03 \mathrm{eV}$ has been removed from the experimental value); $R_{0}$ is the equilibrium bond length (in $\AA$ ); and $\omega_{e}$ is the harmonic vibrational frequency (in $\mathrm{cm}^{-1}$ ). Unless otherwise indicated, the ANO-QZ basis (see text) is used. CBS extrapolation of $D_{e}$ is shown also, when applicable.

\begin{tabular}{|c|c|c|c|c|}
\hline Method & $D_{e}$ & $D_{e}(\mathrm{CBS})$ & $R_{0}$ & $\omega_{e}$ \\
\hline \multicolumn{5}{|l|}{ Multireference perturbation theory } \\
\hline PT2-NEV (larger basis) $)^{\mathrm{a}, \mathrm{b}, \mathrm{c}}$ & 4.8845 & $\ldots$ & 1.9187 & 507.64 \\
\hline PT2-NEV ${ }^{\mathrm{a}, \mathrm{b}, \mathrm{d}}$ & 5.055 & $\ldots$ & 1.9198 & 506.09 \\
\hline PT3-NEV ${ }^{\mathrm{a}, \mathrm{d}, \mathrm{e}}$ & 3.9868 & $\ldots$ & 1.9500 & 461.54 \\
\hline $\mathrm{PT} 2^{\mathrm{f}, \mathrm{g}}$ & 4.41 & $\ldots$ & 1.950 & 459 \\
\hline $\mathrm{MRSDCI}+Q^{\mathrm{h}}$ & 3.9 & $\ldots$ & 1.993 & 447.5 \\
\hline $\operatorname{CCSD}(\mathrm{T})$ & 3.85 & 4.06 & 1.913 & 549 \\
\hline AFQMC/UHF & $4.45(1)$ & $4.66(1)$ & $1.955(4)$ & $428(5)$ \\
\hline AFQMC/CASSCF & $4.20(5)$ & $4.46(5)$ & $1.95(2)$ & $467(24)$ \\
\hline Experiment & & $4.51(1)^{\mathrm{i}}$ & $1.940(9)^{\mathrm{j}}$ & $477.1^{\mathrm{k}}$ \\
\hline
\end{tabular}

\footnotetext{
${ }^{\mathrm{a}}$ Ref. 2.

${ }^{\mathrm{b}}$ Strongly contracted NEVPT2(12e,12o) method.

${ }^{\mathrm{c}}$ Basis set: full ANO basis (10s9p9d6f4g2h).

${ }^{\mathrm{d}}$ Basis set: ANO-QZ basis without the h functions $(8 \mathrm{~s} 7 \mathrm{p} 5 \mathrm{~d} 3 \mathrm{f} 2 \mathrm{~g})$

e Strongly contracted NEVPT3(12e,12o) method.

${ }^{\mathrm{f}}$ Ref. 3 .

${ }^{\mathrm{g}}$ CASPT2(12e,12o) method.

${ }^{\mathrm{h}}$ Ref. 1. Calculations use an ECP with $5 \mathrm{~s} 5 \mathrm{p} 4 \mathrm{~d} 1 \mathrm{f}$ basis (see cited article for detail).

${ }^{\mathrm{i}}$ Ref. 25.

${ }^{j}$ Ref. 26.

${ }^{\mathrm{k}}$ Ref. 27.
} 
TABLE II. Excited state $\left(a^{3} \Sigma_{u}^{+}\right)$spectroscopic properties of $\mathrm{Mo}_{2}$ computed using phaseless AFQMC and other quantum chemistry methods. $T_{e}$ is the excitation energy from the ground state (in units of eV); $R_{0}$ is the bond length at the PEC minimum (in $\AA$ ); and $\omega_{e}$ is the harmonic vibrational frequency (in $\mathrm{cm}^{-1}$ ). Unless otherwise indicated, the ANO-QZ basis (see text) is used. CBS extrapolation of $T_{e}$ is shown also, when applicable.

\begin{tabular}{lcccl}
\hline \hline Method & $T_{e}$ & $T_{e}(\mathrm{CBS})$ & \multicolumn{1}{c}{$R_{0}$} & \multicolumn{1}{c}{$\omega_{e}$} \\
\hline $\mathrm{PT}^{\mathrm{a}, \mathrm{b}}$ & 1.105 & $\ldots$ & 2.063 & 393 \\
AFQMC/CASSCF & $1.15(6)$ & $1.15(6)$ & $2.05(1)$ & $399(20)$ \\
Experiment & & $0.9947^{\mathrm{c}}$ & $\ldots$ & $393.7^{\mathrm{c}}$ \\
\hline
\end{tabular}

${ }^{\mathrm{a}}$ Ref. 3 .

${ }^{\mathrm{b}}$ CASPT2(12e,12o) method.

${ }^{\mathrm{c}}$ Ref. 28.

\section{DISCUSSION}

In this section, we analyze in more detail both the comparison of AFQMC with other methods and the comparison of the correlation effects in $\mathrm{Mo}_{2}$ with $\mathrm{Cr}_{2}$. Correlation effects are greatly reduced in $\mathrm{Mo}_{2}$ compared to $\mathrm{Cr}_{2}$. While the CASSCF result is not bound for $\mathrm{Cr}_{2}$ for an active space as large as $(12 \mathrm{e}, 28 \mathrm{o})$, in $\mathrm{Mo}_{2} \mathrm{CASSCF}(12 \mathrm{e}, 12 \mathrm{o})$ already recovers $30 \%$ of the binding energy. For systems with strong static correlation, multireference perturbation theory has often been the method of choice. As shown in Table I, however, the results depend rather sensitively on the perturbative implementation. Given that $\mathrm{Mo}_{2}$ is considerably more benign than $\mathrm{Cr}_{2}$, it is perhaps somewhat surprising that it turns out to be rather challenging to the best quantum chemistry methods.

\section{A. Comparison with other theoretical results}

The CASPT2 calculation of Borin et al., ${ }^{3}$ using the ANO-QZ basis and a CASSCF $(12 \mathrm{e}, 12 \mathrm{o})$ active space zeroorder wave function, overestimates $D_{e}$ by $\sim 0.2 \mathrm{eV}$. In $\mathrm{Cr}_{2}$, CASPT2 $(12,12)$ shows much larger overbinding of $\sim 1.0 \mathrm{eV}$, based on direct comparisons with the exact FP-AFQMC. ${ }^{15}$ This trend has been attributed to the inadequacy of the active space chosen for the zeroth-order wave function., ${ }^{2,20}$ In $\mathrm{Cr}_{2}$, an improved zeroth-order wave function was obtained for a larger active space of 12 electrons and 28 orbitals, using a self-consistent density matrix renormalization group (DMRG) calculation. ${ }^{20}$ The subsequent CASPT2 calculation ${ }^{20}$ reduces, but does not eliminate, the discrepancy with exact FP-AFQMC, resulting in underbinding by $\sim 0.4 \mathrm{eV}^{15}$ (See also Ref. 23 for a treatment of $\mathrm{Cr}_{2}$ with a split partitioning of the active space.) Multireference perturbative methods are also sensitive to the perturbative implementation. Results from an alternative perturbation treatment, using $n$-electron valence perturbation theory (NEVPT) ${ }^{24}$ are also shown in Table I. The second-order PT2-NEV results show larger overbinding, while third-order PT3-NEV is underbound by $\sim 0.2 \mathrm{eV}$. The $D_{e}$ of PT2-NEV is reduced by $0.2 \mathrm{eV}$ upon increasing the ANO basis size. This is opposite to AFQMC and $\operatorname{CCSD}(\mathrm{T})$, which show $D_{e}$ increasing with basis size. The basis convergence of the perturbative calculations does not follow the empirical $x^{-3}$ behavior, where $x$ is the (correlation consistent) basis cardinal number. (Hence no CBS extrapolation is performed on the results, as mentioned earlier.) In view of these considerations, the agreement of the CASPT2 $\mathrm{Mo}_{2}$ ANO-QZ calculation with the experimental binding energy is likely somewhat fortuitous.

Table I also presents results from multireference singles and doubles configuration interaction (MRSDCI) calculations, ${ }^{1}$ with $Q$ correction applied, and from $\operatorname{CCSD}(\mathrm{T})$. The MRSDCI $+Q$ used an ECP and a smaller basis. Singlereference $\operatorname{CCSD}(\mathrm{T})$, with restricted $\mathrm{HF}$ reference wave function, performs much better in $\mathrm{Mo}_{2}$ than in $\mathrm{Cr}_{2}$. While $\operatorname{CCSD}(\mathrm{T})$ predicts that the $\mathrm{Cr}_{2}$ molecule is unbound, for $\mathrm{Mo}_{2}$ it yields respectable agreement with experiment. Nevertheless, the multireference character of the $\mathrm{Mo}_{2}$ ground state is sufficiently strong that $\operatorname{CCSD}(\mathrm{T})$ still underestimates $D_{e}$ by $0.45 \mathrm{eV}$, as shown in Table I.

For the $\mathrm{Mo}_{2}$ ground state, all of the standard methods have difficulty obtaining an accurate dissociation energy. For the triplet excited state (Table II), we find that the AFQMC and CASPT2 results agree very well with each other and with the experiment.

\section{B. Comparing $\mathrm{Cr}_{2}$ and $\mathrm{Mo}_{2}$}

In this section, we quantitatively compare the effect of electron-electron correlation in $\mathrm{Cr}_{2}$ and $\mathrm{Mo}_{2}$. Under the frozen-core Hamiltonian, these molecules have the same number of correlated electrons, so that the correlation energies are directly comparable. We use the exact FPAFQMC to benchmark the relative effects between the two molecules. Figure 3 compares the magnitude of electronelectron correlation effects in the $\mathrm{Cr}_{2}$ and $\mathrm{Mo}_{2}$ molecules. Results for $\mathrm{Cr}_{2}$ were obtained using the cc-pwCVTZ-DK basis at the experimental bond length $R=1.6788 \AA$ (see Ref. 15); the $\mathrm{Cr}_{2}$ RHF energy is $-2098.533662 E_{\mathrm{h}}$. Results for $\mathrm{Mo}_{2}$ were obtained using the ANO-QZ basis near the experimental bond length $R=1.9 \AA$; the $\mathrm{Mo}_{2}$ RHF energy is $-8091.069911 E_{\mathrm{h}}$. Although the correlation energy is $\sim 0.5 E_{\mathrm{h}}$ larger in $\mathrm{Cr}_{2}$ than in $\mathrm{Mo}_{2}$, the $\mathrm{Cr}_{2} \mathrm{UHF}$ wave function recovers a larger fraction of the correlation energy, $32 \%$, versus $15 \%$ in $\mathrm{Mo}_{2}$. The CASSCF wave function shows a similar but less pronounced trend, recovering 39\% and 28\% in $\mathrm{Cr}_{2}$ and $\mathrm{Mo}_{2}$, respectively.

The stronger correlation effects in $\mathrm{Cr}_{2}$, however, are evident in the top panel of Fig. 3(b): achieving 95\% of the CASSCF total wave function weight requires retaining 559 determinants in $\mathrm{Cr}_{2}$ but only 168 in $\mathrm{Mo}_{2}$. This is also evident in the larger $\operatorname{CCSD}(\mathrm{T})$ discrepancy in $\mathrm{Cr}_{2}$ than in $\mathrm{Mo}_{2}, \sim 40 \mathrm{~m} E_{\mathrm{h}}$ and $\sim 7 \mathrm{~m} E_{\mathrm{h}}$, respectively. The dependence of the AFQMC/CASSCF energies on the quality of the trial wave function is shown in the bottom panel of Fig. 3(b). The dependence is significantly stronger in $\mathrm{Cr}_{2}$, where at 95\% cutoff, the total energy is still $\sim 13 \mathrm{~m} E_{\mathrm{h}}$ higher than the exact value (this error is $\sim 5 \mathrm{~m} E_{\mathrm{h}}$ for $\mathrm{Mo}_{2}$ ). At the variational level, although the truncated wave function recovers more correlation energy in $\mathrm{Cr}_{2}$ than in $\mathrm{Mo}_{2}$ for the same weight cut, its performance is worse in AFQMC/CASSCF. We attribute this to the larger dynamic correlation energy that must be recovered in $\mathrm{Cr}_{2}$. Cancellation of errors between the molecule and atom AFQMC energies leads to better agreement in binding energy, however. For the best truncated CASSCF 

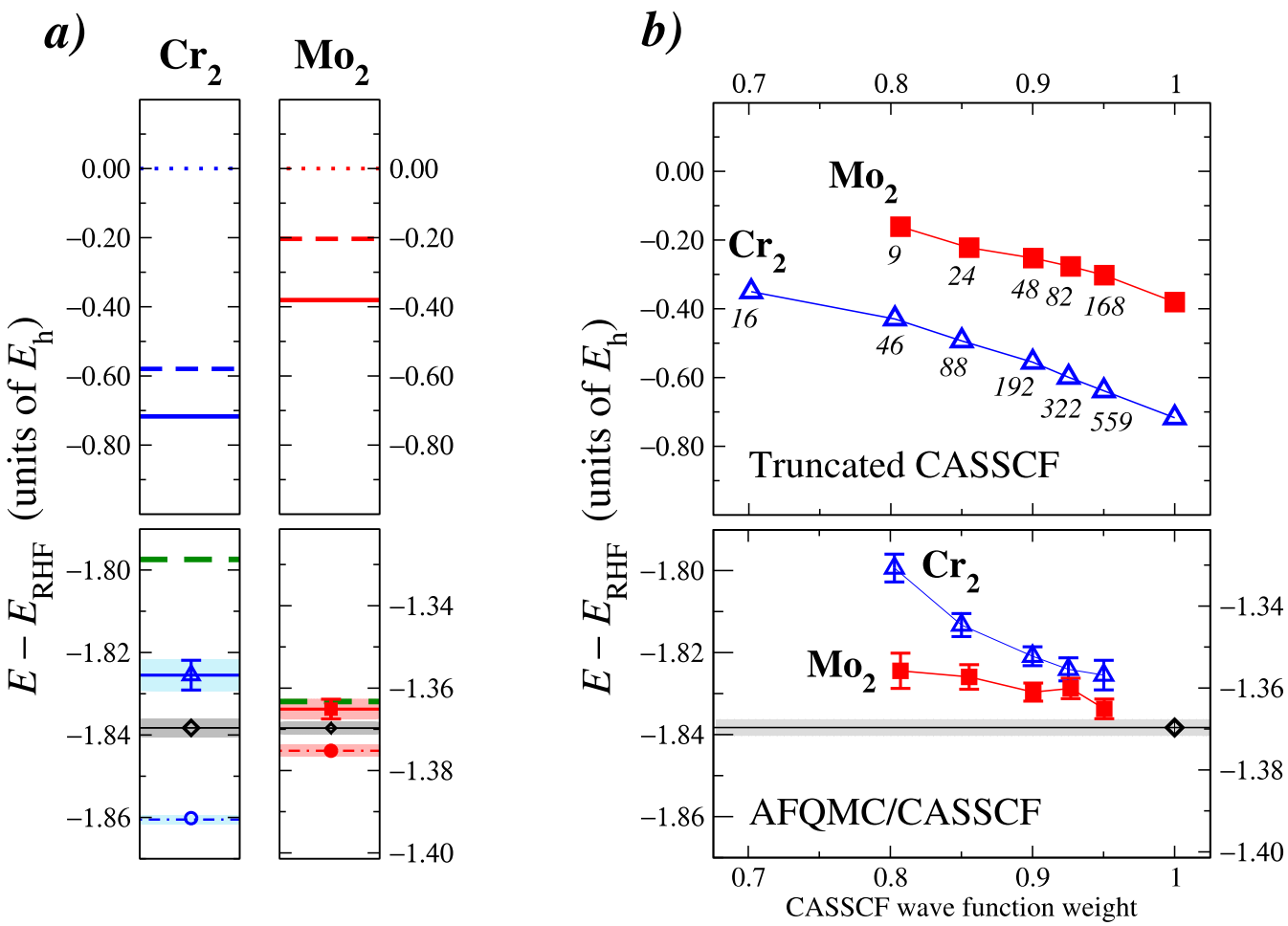

FIG. 3. The correlation energy in the $\mathrm{Cr}_{2}$ and $\mathrm{Mo}_{2}$ molecules. All energies are reported relative to the restricted $\mathrm{HF}$ (RHF) energy. Note that the energy offset in the lower panels of (a) and (b) aligns the (exact) FP-AFQMC correlation energies of $\mathrm{Cr}_{2}$ and $\mathrm{Mo}_{2}$. (a) Upper panels: RHF (dotted zero baseline), UHF (dashed line), and CASSCF (solid line) energies. Lower panels: CCSD(T) (dashed line), AFQMC/CASSCF at 95\% weight cutoff (triangle and square), FP-AFQMC (diamond), AFQMC/UHF (circle); the AFQMC statistical uncertainties are indicated by the shading. (b) Correlation energy as a function of the retained weight in the multi-determinant CASSCF wave function. Solid squares and open triangles denote the $\mathrm{Mo}_{2}$ and $\mathrm{Cr}_{2}$ results, respectively. Upper panel: variational energy of the truncated CASSCF wave function; numbers adjacent to the symbols give the number of determinants in the truncated wave function. Lower panel: the corresponding AFQMC/CASSCF correlation energies; statistical uncertainties indicated by the error bars. The exact FP-AFQMC energy from (a) is also shown. See the text for additional details.

wave function, the error in the binding energy is $\sim 5 \mathrm{~m} E_{\mathrm{h}}$ $(\sim 0.14 \mathrm{eV})$ for $\mathrm{Cr}_{2}$, and virtually exact for $\mathrm{Mo}_{2}$.

Basis set errors will modify the correlation energy recovered by the different methods. The mean-field HF energies are quite well converged for the basis sets used here. For the many-body calculations, we estimate (using $\mathrm{AFQMC} / \mathrm{UHF}$ ) the CBS shifts to be $\sim-100 \mathrm{~m} E_{\mathrm{h}}$ and $\sim-70 \mathrm{~m} E_{\mathrm{h}}$ for $\mathrm{Cr}_{2}$ and $\mathrm{Mo}_{2}$, respectively. For the purpose of the above comparisons, however, the relative error between the various approximate and exact methods should not change significantly.

\section{SUMMARY}

We have presented an accurate calculation of the $\mathrm{Mo}_{2}$ ground state $\left(X^{1} \Sigma_{g}^{+}\right)$and first triplet excited state $\left(a^{3} \Sigma_{u}^{+}\right)$. We use the phaseless AFQMC method with the truncated CASSCF trial wave function (AFQMC/CASSCF). Calculations were done using high-quality, realistic basis sets, and extrapolation to the CBS limit is performed. The resulting PECs and spectroscopic constants are in excellent agreement with experiment. Comparisons are made with other high-level quantum chemistry methods. We have also quantified the extent of strong electron correlations in both $\mathrm{Cr}_{2}$ and $\mathrm{Mo}_{2}$ molecules. Molybdenum is important in a variety of systems which can potentially exhibit exotic properties from strong correlation and topological effects. Our results can serve as a benchmark as theoretical and computational methods are developed and employed to treat such systems reliably.

\section{ACKNOWLEDGMENTS}

This work was supported by DOE (Grant No. DE-FG0209ER16046), NSF (Grant No. DMR-1409510), and ONR (Grant No. N000141211042). We acknowledge a DOE CMCSN Grant (No. DE-FG02-11ER16257) for facilitating stimulating interactions. An award of computer time was provided by the Innovative and Novel Computational Impact on Theory and Experiment (INCITE) program, using resources of the Oak Ridge Leadership Computing Facility (Titan) at the Oak Ridge National Laboratory, which is supported by the Office of Science of the U.S. Department of Energy under Contract No. DE-AC05-00OR22725. We also acknowledge the computational support provided by William and Mary High Performance Computing facility. The authors would like to thank Valera Veryazov for clarifying the ANO basis, and Fengjie Ma and Hao Shi for many useful discussions.

${ }^{1}$ K. Balasubramanian and X. Zhu, J. Chem. Phys. 117, 4861 (2002).

${ }^{2}$ C. Angeli, A. Cavallini, and R. Cimiraglia, J. Chem. Phys. 127, 074306 (2007).

${ }^{3}$ A. C. Borin, J. P. Gobboa, and B. O. Roos, Chem. Phys. 343, 210 (2008).

${ }^{4}$ S. Zhang and H. Krakauer, Phys. Rev. Lett. 90, 136401 (2003).

${ }^{5}$ W. A. Al-Saidi, S. Zhang, and H. Krakauer, J. Chem. Phys. 124, 224101 (2006). 
${ }^{6}$ M. Suewattana, W. Purwanto, S. Zhang, H. Krakauer, and E. J. Walter, Phys. Rev. B 75, 245123 (2007).

${ }^{7}$ S. Zhang, in Emergent Phenomena in Correlated Matter, edited by E. Pavarini, E. Koch, and U. Schollwöck (Forschungszentrum Jülich Zentralbibliothek, Verlag Jülich, 2013), Chap. 15, ISBN: 3893368841, http://juser. fz-juelich.de/record/137827.

${ }^{8}$ W. Purwanto, H. Krakauer, and S. Zhang, Phys. Rev. B 80, 214116 (2009). ${ }^{9}$ H. Shi and S. Zhang, Phys. Rev. B 88, 125132 (2013).

${ }^{10}$ S. Zhang, J. Carlson, and J. E. Gubernatis, Phys. Rev. B 55, 7464 (1997).

${ }^{11}$ W. Purwanto and S. Zhang, Phys. Rev. E 70, 056702 (2004).

${ }^{12}$ W. Purwanto and S. Zhang, Phys. Rev. A 72, 053610 (2005).

${ }^{13}$ W. Purwanto, S. Zhang, and H. Krakauer, J. Chem. Phys. 130, 094107 (2009).

${ }^{14}$ Y. Virgus, W. Purwanto, H. Krakauer, and S. Zhang, Phys. Rev. Lett. 113, 175502 (2014).

${ }^{15}$ W. Purwanto, S. Zhang, and H. Krakauer, J. Chem. Phys. 142, 064302 (2015).

${ }^{16}$ F. Ma, W. Purwanto, S. Zhang, and H. Krakauer, Phys. Rev. Lett. 114, 226401 (2015).

${ }^{17}$ H. Shi, C. A. Jiménez-Hoyos, R. Rodríguez-Guzmán, G. E. Scuseria, and S. Zhang, Phys. Rev. B 89, 125129 (2014).
${ }^{18}$ W. Purwanto, H. Krakauer, Y. Virgus, and S. Zhang, J. Chem. Phys. 135, 164105 (2011).

${ }^{19}$ B. O. Roos, R. Lindh, P.-Å. Malmqvist, V. Veryazov, and P.-O. Widmark, J. Phys. Chem. A 109, 6575 (2005).

${ }^{20}$ Y. Kurashige and T. Yanai, J. Chem. Phys. 135, 094104 (2011).

${ }^{21}$ A. Halkier, T. Helgaker, P. Jorgensen, W. Klopper, and J. Olsen, Chem. Phys. Lett. 302, 437 (1999).

${ }^{22}$ T. Helgaker, W. Klopper, H. Koch, and J. Noga, J. Chem. Phys. 106, 9639 (1997).

${ }^{23}$ G. L. Manni, D. Ma, F. Aquilante, J. Olsen, and L. Gagliardi, J. Chem. Theory Comput. 9, 3375 (2013).

${ }^{24}$ C. Angeli, R. Cimiraglia, and J.-P. Malrieu, J. Chem. Phys. 117, 9138 (2002).

${ }^{25}$ B. Simard, M.-A. Lebeault-Dorget, A. Marijnissen, and J. J. ter Meulen, J. Chem. Phys. 108, 9668 (1998).

${ }^{26}$ J. B. Hopkins, P. R. R. Langridge-Smith, M. D. Morse, and R. E. Smalley, J. Chem. Phys. 78, 1627 (1983).

${ }^{27}$ Y. M. Efremov, A. N. Samoilova, V. B. Kozhukhovsky, and L. V. Gurvich, J. Mol. Spectrosc. 73, 430 (1978).

${ }^{28}$ D. Kraus, M. Lorenz, and V. E. Bondybey, PhysChemComm 4, 44 (2001). 